G443(P) FOLLOW-UP BETWEEN 6 AND 24 MONTHS AFTER DISCHARGE FROM TREATMENT FOR SEVERE ACUTE MALNUTRITION IN CHILDREN AGED 6-59 MONTHS: A SYSTEMATIC REVIEW

N O'Sullivan, M Kerac, P James. Nutrition for Global Health, London School of Hygiene and Tropical Medicine, London, UK

10.1136/archdischild-2018-rcpch.432

Background Severe acute malnutrition (SAM) is a major global health problem estimated to affect 16.9 million children under-5, and confers an increased risk of mortality and morbidity. Community Management of Acute Malnutrition (CMAM) programmes have revolutionised treatment for SAM and significantly reduced associated mortality. Community follow-up after discharge from treatment is variable, as links to supplementary feeding programmes are often weak. This review aimed to draw attention to the uniquely vulnerable period of 6-24 months post-discharge that, until now, has fallen through the gap.

Methods Embase, Global Health and MEDLINE were systematically searched with terms related to SAM, nutritional intervention and follow-up between June and August 2017.

Results A total of 3691 articles were retrieved from the search, 55 full-texts were screened and seven met the inclusion criteria to be included in the review. Loss-to-follow-up, mortality, relapse, morbidity and anthropometry were outcomes reported. Between $45.1 \%$ and $0.0 \%$ of cohorts were lost-to-follow-up. Mortality ranged from $10.4 \%$ to $0.6 \%$ at an average of 12 months after-discharge. Two studies reported improvement of weight-for-height (WHZ) from discharge to 12 month follow-up, whilst the three studies that reported height-for-age (HAZ) found either limited or no improvement. Conclusion This review suggests there is a scarcity of studies that follow-up children for at least 6 months after-discharge. Therefore, there is an urgent need for further follow-up studies investigating a diverse range of outcomes, using control groups and streamlined admission and discharge criteria.

This review has demonstrated that children remain vulnerable to adverse outcomes after discharge from SAM treatment programmes. We must begin to look beyond anthropometric definitions of recovery and the sole focus of reducing mortality. Instead, we should take a holistic view of child nutrition that considers the role of the home and community environment for a complete rehabilitation of these children, and uses CMAM as an opportunity to promote good nutrition and nutritional practices in children for life.

\section{G444(P) MEDICAL STUDENTS EMBRACING CHANGE: HOW DO WE LEARN FROM LESSONS OF THE PAST TO BE GREAT DOCTORS OF THE FUTURE?}

Z Vickers, H Avula, CR Fertleman. Medicine, University College London, London, UK

10.1136/archdischild-2018-rcpch.433

In order to improve clinical practice in the future we have to learn from our past. The issues discussed at the inaugural Great Ormond Street Hospital Advances in Paediatrics Conference 2017 were current and pressing and should be dealt with now to raise the bar of healthcare in the UK. As medical students, we considered the salient messages from this conference and discuss how to apply them to our future training.
Staff were frank and honest about where paediatric medicine was heading. They alluded to mistakes made allowing for reflection and open discussion, whilst suggesting changes we hope would make us more rounded paediatricians.

Key themes that shaped the inquisitive, open mind-set that medical students should have throughout their training and beyond were highlighted. Three points seemed especially important: having a realistic perspective on the 'crisis of the NHS', awareness of the lack of transparency in clinical research and the advancement of technology and associated impact on patient-centred care. If we are able to address some of these issues it might help to not only maintain but also raise the standards of healthcare that we can provide.

Overall, we feel that by embracing change, especially at the beginnings of our education, we can overcome miscalculations made by our predecessors. It is easier to have the right attitude ingrained in you from the start than it is to alter one already set in stone. By shifting focus to patient-centred care we can put patients and families back in the driving seat of their own health. Changes supporting openness in clinical trials approach can ensure that patients are not harmed by poor clinical decisions based on research misconduct and publication bias. To be great future doctors we must be receptive to changes going on around us. Technology is revolutionising medicine; we should embrace it and adapt it into our practice to provide the best care. As Professor Tony Young-Head of NHS Innovation England-said, this will enable the clinicians of now and the future to be 'advisors and navigators' and not just 'gatekeepers to medicine.'

\section{British Paediatric Respiratory Society and British Society for Paediatric and Adolescent Rheumatology}

\section{G445 PHYSICAL AND PSYCHOLOGICAL ADAPTATION TO IMPAIRED LUNG FUNCTION IN CHILDREN AND YOUNG PEOPLE WITH SCOLIOSIS}

${ }^{1} \mathrm{~F}$ Jagger, ${ }^{2} \mathrm{~A}$ Tsirikos, ${ }^{1} \mathrm{D}$ Urquhart. ${ }^{1}$ Department of Respiratory Medicine, Royal Hospital for Sick Children, Edinburgh, UK; ${ }^{2}$ Scottish National Spine Deformity Centre, Royal Hospital for sick Children, Edinburgh, UK

\subsection{6/archdischild-2018-rcpch.434}

Aims Scoliosis may impair both lung function and exercise capacity. There is some evidence to suggest lung function is positively correlated with exercise capacity, however the exact relationship between these variables is poorly defined. This study investigated the relationship between exercise variables and lung function in children and young people with scoliosis, looking particularly at whether patients show evidence of improved physical conditioning despite impaired lung function. Methods Retrospective analysis of data from Spirometry [FEV1, FVC], Whole Body Plethysmography, Cardiopulmonary Exercise Testing [CPET] and patient outcome questionnaires (SRS-22) undertaken prior to spinal fusion. CPET measures included maximal exercise capacity [VO2peak] as well as $\mathrm{VO} 2$ at anaerobic threshold [AT] expressed as\%predicted VO2maxa measure of physical conditioning, and minute ventilation [VE] from which breathing reserve [BR] could be calculated. Analyses were performed using SPSSv23, and correlations expressed using Pearson's correlation co-efficient. 